\title{
TWO-ELECTRON QUANTUM DOTS IN MAGGNTIC FIELD
}

\author{
J. Adamowski and B. Spisak \\ Faculty of Physics and Nuclear Techniques, Technical University (AGH) \\ Al. Mickiewicza 30, 30-059 Kraków, Poland
}

\begin{abstract}
A theoretical description is given for electronic properties of semiconductor quantum dots in a magnetic field. A two-electron model is applied for electrons in a cylindrical quantum dot with a parabolic confinement potential. The eigenvalue problem is solved by the variational method with the trial wave function proposed in the form of linear combination of $S$-type and $P$-type Gaussians. The energy levels of singlet and triplet states with arbitrary radial and magnetic quantum numbers have been calculated as a function of the applied magnetic field. The calculated cyclotron transition energies agree well with those measured for InGaAs/GaAs quantum dots. It is shown that the electron-electron interaction has a small influence on the transition energy.
\end{abstract}

PACS numbers: 73.20.Dx

Quantum dots (QDs) are artificially made nanostructures of dimensions 1-100 nm. They consist of a finite number (from 1 to about 100) of electrons, which are not bound with ions, but are confined within the QD region. Recently, the QDs of many different shapes are fabricated. The most common are those of cylindrical and spherical forms. The cylindrically shaped QDs embedded in semiconductor layer structures are investigated in Refs. [1,2]. They are designed in order to get miniaturized field-effect-transistors $[1,2]$. The nanostructure studied in Ref. [2] is built from a sequence of layers of lateral diameter of about $20 \mathrm{~nm}$ and consists of a semiinsulating GaAs substrate covered by a strongly doped GaAs buffer layer, an undoped GaAs layer, on top of which is a few-monolayer thick $\mathrm{In}_{0.5} \mathrm{Ga}_{0.5} \mathrm{As}$ quantum dot, which is embedded in a $5 \mathrm{~nm}$ thick GaAs layer. The blocking barrier is formed by a $30 \mathrm{~nm}$ thick AlAs/GaAs superlattice with a period of $4 \mathrm{~nm}$. The nanostructure is covered by a GaAs cap and a semitransparent $\mathrm{NiCr}$ gate electrode. The authors of Ref. [2] measured the infrared transmission spectra in the magnetic field, which was applied in the growth direction. They recorded the cyclotron transition energy and interpreted their results with the help of the one-electron model.

There arises a problem of the influence of the electron-electron interaction on the energy spectrum and transition energy. The present paper provides the 
solution to this problem in the frame of the two-electron model, which allows us to treat accurately the electron-electron interaction preserving the main qualitative properties of the interacting many-electron system. Our purpose is to solve the eigenvalue equation for the two electrons in a parabolic confinement potential and external magnetic field and find the transition energy between the low-energy quantum states.

The QD of the very small thickness [2] can be treated as a quasi-two-dimensional system of a cylindrical symmetry. We apply the effective-mass envelope-function method and assume the parabolic form of the confinement potential acting on the electron, i.e. $V_{\text {conf }}\left(r_{i}\right)=m_{\mathrm{e}} \omega_{0}^{2} r_{i}^{2} / 2$, where $m_{\mathrm{e}}$ is the effective conduction-band mass of the electron, $\omega_{0}$ is the "confinement frequency", and $r_{i}=\left(x_{i}, y_{i}, 0\right)$, $i=1,2$. The external static uniform magnetic field is applied in the growth $(z)$ direction, i.e. $B=(0,0, B)$. We introduce the following units and parameters: unit of length ("confinement length") $a_{0}=\left(\hbar / m_{\mathrm{e}} \omega_{0}\right)^{1 / 2}$, unit of energy $\hbar \omega_{0}$, strength of electron-electron repulsion $\lambda=a_{0} / a_{B}$, where $a_{B}=\varepsilon_{0} \hbar^{2} / m_{\mathrm{e}} e^{2}$ and $\varepsilon_{0}$ is the static dielectric constant, and frequency ratio $\mu=\omega_{\mathrm{c}} / \omega_{0}$, where $\omega_{\mathrm{c}}=e B / m_{\mathrm{e}}$ is the cyclotron frequency. The Hamiltonian $H$ of the two-electron system in the confinement potential $V_{\text {conf }}$ and magnetic field $\boldsymbol{B}$ can be separated in the center-of-mass and relative coordinates. In the present paper, we introduce the following position vectors: $\boldsymbol{R}=\left(r_{1}+r_{2}\right) / \sqrt{2}$ and $r=\left(r_{1}-r_{2}\right) / \sqrt{2}$, which leads to the separation $H=H_{R}+H_{r}$, where

$$
H_{r}=-\frac{1}{2} \nabla_{r}^{2}+\frac{\mu}{2} L_{z}+\frac{1}{2}\left(1+\frac{\mu^{2}}{4}\right) r^{2}+\frac{\lambda}{\sqrt{2} r}
$$

and $H_{R}$ has the same form, but without the last term. In Eq. (1), $L_{z}$ is the $z$-component angular momentum operator. The eigenvalue problem for $H_{R}$ possesses the well-known Fock-Darwin [3] solutions, on which the one-electron model [2] is based. Several numerical methods [4-6] were used in order to solve the eigenvalue problem for Hamiltonian (1). Recently, Zhu et al. [7] have applied the method of series expansion with different series in different regions of space. In the present paper, we propose the variational method with the Gaussian basis wave functions. This approach is quite simple in programming, leads to the results of comparable accuracy to those in Refs. [4-7], and allows us to calculate the energy levels and other experimentally accessible quantities for the singlet and triplet states of arbitrary radial and magnetic quantum numbers.

The eigenstates and eigenvalues of $H_{R}$ and $H_{r}$ are labelled by the radial $(N$ and $n$ ) and magnetic $(M$ and $m$ ) quantum numbers. The energy eigenvalues of the total Hamiltonian $H$ are given by

$$
E_{N M n m}=E_{N M}+E_{n m} \text {, }
$$

where the energy levels of the Hamiltonians $H_{R}$ and $H_{r}$ are given by the first and the second term in the right-hand side, respectively. Both solutions are identical for $\lambda=0$, i.e., without the electron-electron interaction. In this paper we neglect the spin-splitting of energy levels in the magnetic field, which is not observed in the experiments $[1,2]$. The eigenstates of Hamiltonian (1) can be classified according to the total spin of the two-electron system; the spin singlet states are associated with the symmetric spatial wave functions, i.e., these with even $m$, and the spin triplet states - the antisymmetric spatial wave functions, i.e., $m-$ odd. 
For $r \rightarrow 0$ and $m=0$, we obtain the following analytical (unnormalized) form of the ground-state wave function: $\psi(r) \simeq 1+2 \lambda r$, which is in agreement with the cusp condition for electrons. In a general case, we solve the eigenvalue equation for Hamiltonian (1) by the variational method, with the trial wave function being a linear combination of $S$-type Gaussians, $\exp \left(-\alpha_{s} r^{2}\right)$, and $P$-type Gaussians, $r \exp \left(-\alpha_{p} r^{2}\right)$, where $\alpha_{s, p}$ are the nonlinear variational parameters. We apply the mixed $S$-type and $P$-type basis for the states with $m=0$, and the $P$-type basis for the states with $m \neq 0$. Very accurate results for the low-energy levels are obtained with 10 basis functions.

Figure $1 \mathrm{~A}$ shows the calculated energy levels $E_{n m}$ as a function of cyclotron frequency. The shapes of curves in Fig. 1A qualitatively agree with those measured by the single-electron capacitance spectroscopy [1] for GaAs quantum dots, which yields a justification of the assumed parabolic form of the confinement potential.
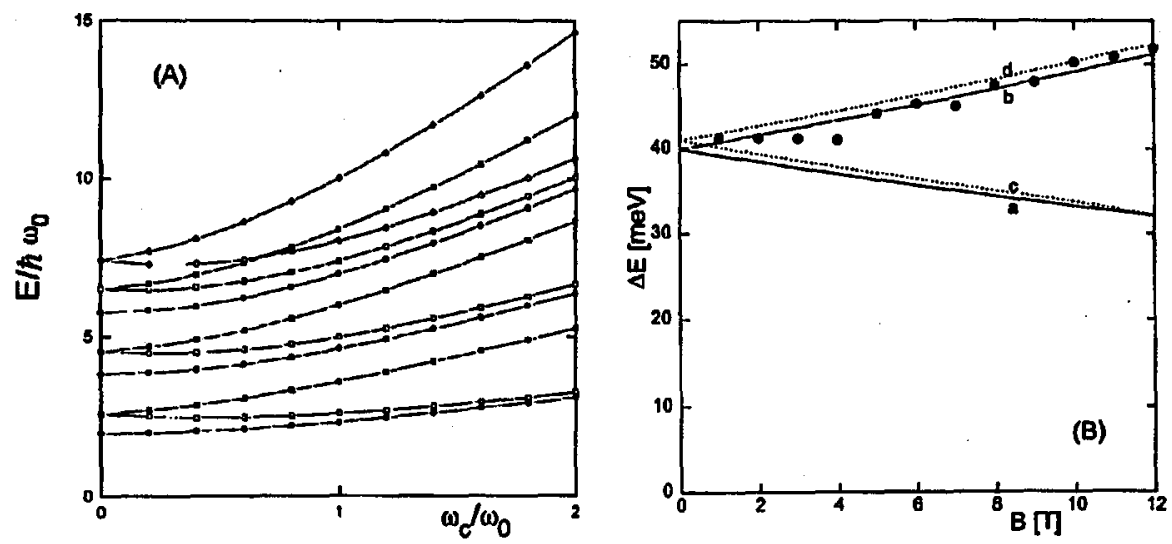

Fig. 1. (A) Calculated energy spectrum $E_{n m}$ of the two-electron relative-coordinate Hamitonian vs. magnetic field. Curves with full circles correspond to $m=0$, open squares $-m=-1$, full squares $-m=1$, open diamonds $-m=-2$, and full diamonds $-m=2$. The radial quantum number $n=0,1,2$ for the subsequent levels, the electron-electron repulsion strength $\lambda=1, \omega_{c}$ is the cyclotron frequency, and $\omega_{0}-$ confinement frequency. (B) Transition energy between the ground state $(0,0)$ and the excited states $(0,-1)$ (curves (a) and (c)) and (0,1) (curves (b) and (d)) as a function of the magnetic field. Curves (a) and (b) ((c) and (d)) correspond to the two-electron (one-electron) model, and the dots are experimental points.

We have applied our approach to the QD in a magnetic field, for which the magnetooptical transmission measurements [2] were performed. The calculated and measured [2] cyclotron transition energies are displayed in Fig. 1B. Since the confinement frequency $\omega_{0}$ is an unknown parameter, we treat it as an adjustable parameter and deduce its value from the fit to the experimental data [2]. In the two-electron model, we obtain $\hbar \omega_{0}=40 \mathrm{meV}$, while the one-electron model [2] leads to $\hbar \omega_{0}=41 \mathrm{meV}$. The values of the other parameters are taken on to be 
the same as in Ref. [2], i.e. $m_{\mathrm{e}}=0.07 m_{\mathrm{e} 0}$ and $\varepsilon_{0}=13.0$. The two pairs of curves in Fig. 1B show the results of the one-electron (broken curves) and two-electron (solid curves) model. The curves (a) and (c) correspond to the $(0,0) \rightarrow(0,-1)$ transitions, which are not observed, since these energies lie in the reststrahlen regime [2]. The curves (b) and (d) correspond to the observed [2] $(0,0) \rightarrow(0,1)$ transitions. The transition energy, calculated in the present paper in the frame of the two-electron model (curve (b)), agree very well with the experimental results [2]. We should note, however, that the correction to the one-electron model (curve (d)) is small. This property is in agreement with the generalized Kohn theorem [8], which states, in the dipole approximation, that the cyclotron transition energy is independent of the electron-electron interaction for the parabolic effective potential. The results of Fig. 1B show that the electron-electron interaction has only a small influence on the transition energy. We note that the present results have been obtained without the use of the dipole approximation for radiative transitions.

A similar problem has been treated by Peeters and Schweigert [9], who additionally included the confinement potential for the electrons in the $z$-direction. These authors [9] approximated the electron-electron interaction by the averaged two-dimensional potential, and obtained the cyclotron transition energies in quite good agreement with the experiment [2] but with a smaller transition energy at the low magnetic field. Due to the uncertainties in the determination of the confinement potential, we can regard both the present results and those of Ref. [9] to be satisfactory.

In future research, it would be interesting to consider in the framework of the present model (or its many-electron generalization) the properties of electrons in quantum dots, which are more strongly dependent on the electron-electron interaction.

\section{References}

[1] R.C. Ashoori, H.L. Stormer, J.S. Weiner, L.N. Pfeiffer, S.J. Pearton, K.W. Baldwin, K.W. West, Phys. Rev. Lett. 68, 3088 (1992).

[2] H. Drexler, D. Leonard, W. Hansen, J.P. Kotthaus, P.M. Petroff, Phys. Rev. Lett. 73, 2252 (1994).

[3] V. Fock, Z. Phys. 47, 446 (1926); C.G. Darwin, Proc. Camb. Phil. Soc. 27, 86 (1930).

[4] U. Merkt, J. Huser, M. Wagner, Phys. Rev. B 43, 7320 (1991).

[5] D. Pfannkuche, V. Gudmundsson, P.A. Maksym, Phys. Rev. B 47, 2244 (1993).

[6] A. Matulis, F.M. Peeters, J. Phys., Condens. Malter 6, 7751 (1994).

[7] J.-L. Zhu, J.-Z. Yu, Z.-Q. Li, Y. Kawazoe, J. Phys., Condens. Matter 8, 7857 (1966).

[8] L. Banyai, S.W. Koch, Semiconductor Quantum Dots, World Scientific, Singapore 1993.

[9] F.M. Peeters, V.A. Schweigert, Phys. Rev. B 53, 1468 (1996). 